\title{
DESIGN AND DEVELOPMENT OF A WEB BASED GEOLOCATION DATABASE FOR THE TELEVISION WHITE SPECTRUM (TVWS) IN NIGERIA.
}

\section{1, Engr. Ezeji Nwamaka.G. 2, Dr Azubogu .A.C.O, 3.Engr. Dr. Nwobodo Nzeribe Nnenna Harmony}

1. Department of Computer Engineering, Enugu State University of Science and Technology, Enugu, Enugu State Nigeria.

2. Department of electronics and computer Engineering, NAU, AwkaAnambra state Nigeria.

3. Department of computer Engineering. Enugu State University of science and Technology Enugu, Enugu State. Nigeria

\section{Abstract}

This research work focused on the development of a web based geo location database tc predicting/finding television white spaces in Ngwo environment in Enugu state, south East Nigeria.

In this thesis low cost spectrum analyzer (RF explorer) was used to identify the unused portion of UHF of the radio spectrum from $470-789 \mathrm{MHz}$ and a geo location database is developed where these unused spectrum were stored for appropriate spectrum management to avoid interference between the licensed and unlicensed users. Afterwards, the developed geo location database is hosted over the internet to be accessed by white space devices. Theses white space devices are the FCC certified wireless devices that can be used without an exclusive broadcast license in the RF spectrum below 700MHz. The WSD will query the database through the web interface and introduces a location, time, day of the week, threshold energy level for that location and the regulatory entity as input parameters. The introduced parameters are sent to the database manager. The database manager will access the database and get the corresponding data about the transmitters in the input location. The TVWS calculation part will use the data from the database and the input parameters to calculate the available free channels for the input location with reference to the standard threshold energy of $-94.5 \mathrm{dBm}$ specified for this work. If the power in the location is lower than this threshold ($94.5 \mathrm{dBm})$, the channel will be considered to be free. Otherwise, when the power is higher than the threshold, the channel will be classified as occupied. The resulting list will be provided to the user through the web interface. The on-line data base was evaluated for Response time: After several measurements, it was concluded that Geo location data base design for this work has an average response time of 1.7 seconds. This was obtained by calculating the average execution time for 10 different queries. The results obtained showed that the developed geo-location database has a response time of 1.7 seconds. From result analysis generated, it was gathered that for a standard energy threshold of $-94.5 \mathrm{dBm}$ specified by FCC in (FCC, 2008), more than 65\% of the frequencies in the UHF band of the television spectrum is left unused at any time of the day and on any day of the week in the test bed area. This TV white space geo-location database is the reliable mechanism to provide in real time the TVWS availability for proper regulatory management in the south eastern part of Nigeria. KEYWORDS: TV white space, white space devices, sensing technique, geo location database. 


\section{INTRODUCTION}

Generally, traditional wireless communication systems have fixed transmission parameters. There transmission frequency is fixed and the same in every location and instant of time determined by regulatory policies, however, the recent popularity of telecommunications and wireless communications has increased the usage of radio spectrum exponentially (Naroa, 2011).

In order to supply all the demand and improve communication parameters and quality of service, new technology needs to be developed .Obviously, an essential response to this increasing demand is the opening of new spectrum both licensed and recently unlicensed (Farzad, 2013). One of the attempts to solve the problem of spectrum lack is cognitive radio (CR) and TV white space (TVWS) communication in particular.

The ongoing global television (TV) broadcast digital switchover (DSO) promises to leave large portions of the very high frequency (VHF, at 174--230 MHz) and ultra-high frequency (UHF, at 470-790 MHz) bands available on a geographical basis for opportunistic use in providing enhanced wireless broadband access. Such spectrum bands are widely known as TV "whitespaces" (TVWS) they are interspersed within 174 - 790 MHZ licensed bands and can allow secondary (unlicensed) users to opportunistically access them provided interference protection guaranteed to the neighboring primary (licensed) networks are ensured (FCC, 2010)(FCC, 2011). The spectrum white spaces are the unused frequencies in a certain geographical area for a reasonable amount of time.

Meanwhile, the spectrum white spaces are identified in television (TV), fixed satellite stations (FSS) and many radar systems (Tizazu, 2012).

While a traditional WI-FI router has a relatively limited range of transmission, which is around 100 meters under perfect conditions, and can be blocked by walls or other environmental barriers TV white space technology can cover about 10 kilometers in diameter which is 100 times the distance of WI-FI. Because of the TV white space superior range and ability to penetrate obstacles such as trees, building and rough terrain it was nicknamed "super WI-FI". (Anderson 2016)

Television White Space refers to the unused TV channels between the active ones in the VHF and UHF frequencies. These unused frequencies are also referred to as buffer channels. In the past these buffers were placed between active TV channels to protect against broadcasting interference. It must be noted at this point that it has since been proven that these unused frequencies can be used to provide broadband internet access while operating harmoniously with surrounding TV channels (Anderson, 2016).

But contrary to the conventional static spectrum allocations, these frequencies are still needed by the primary users (PU) and they cannot be leased for full time. Hence, the secondary users (SU) can use the spectrum only at times when there is no active primary Users. Therefore, an opportunistic access scheme (enabler technology) maybe required for this technique to work efficiently.

The TV white space communications tries to reuse these unused channels by adapting its transmission parameters to the environment and avoid causing interference to the primary users of the TV bands. (Naroa, 2011).

TV white space signals can follow the ground and travel around obstacles and foliage, better signal means less infrastructure making this technology affordable, simple to deploy and friendly on the environment.

It is widely recognized that white space identification is an important mile stone for the wide deployment of next generation wireless network. The propagation characteristics of TVWS are especially good for wireless communication, reducing propagation losses and hence, increasing coverage and very little and relatively cheap infrastructure is required for their 
DOI: https://dx.doi.org/10.26808//rs.ca.i10v2.03

International Journal of Computer Application (2250-1797)

Issue 10 Volume 2, March-April 2020

implementation making them especially suitable for rural and underdeveloped areas or countries. As a result there are two principal use cases of TVWSs (Naroa, 2011).

The first use case is the local communications (so called WI-FI) typically with laptops and hand held, where a wireless access point will offer access to the internet using TVWS frequencies.

The second use case is the wide area broadband in rural areas, with fixed antennas mounted on rooftops. There are also a range of other innovative usages of TVWS that might appear due to its favorable propagation characteristics (Naroa, 2011)

Geo-location database is a centralized store or regional mirror that is used to determine if there are any TVWS (free channels) that can be used without causing interference to other services. ECC need that the WSDs have to recognize and indicate their current location before sending the query.

With this scheme, WSDs are not allowed to transmit before they receive notifications from the database about the available white spaces, if any, in their position. They offer apracticalsolution to monitor capabilities and primary transmissions.

A secondary user has access to a database system to get information about the primary users in a given area. The secondary users interact with the database to know which frequencies are free in a certain time and in a certain location and determine the transmission power they are allowed to use. To offer the white space distribution over the frequency bands, the geolocation database needs some parameters and information such as primary user's frequency of operation, transmitted power, size and type of transmit antenna etc. These parameters are provided to the database system by primary users.

Meanwhile, the main problem of TVWS communication is the possible interference with primary users. Primary user's protection has to be ensured and currently there are two possible techniques to do this:

They are sensing and geo-location database systems, and in this research, geo-location database system approach was used to control interference between licensed and unlicensed users. The geo-location database system is used to determine the available frequencies in a given location and time. This technology is highly preferred by federal communication commission (FCC) and all regulatory bodies. In order to know which channel to use in the TVWSs, white space device (WSDs) access and query a geo-location database system indicating is location and the database system responds with a list of available frequencies and allowed transmit powers for that location. The WSD selects one of the available channels and start its transmission (Carlson Telcodia 2015).

The other technique is the sensing technique which involves detecting radio signals and estimating the relative location of primary users in order not to interfere with them. The approach of spectrum sensing involves the incorporation of a spectrum scanner in all nodes of the secondary wireless network. These scanners/sensors periodically scan the entire TV band for empty (locally unused) channels. For the network to use a specific TV channel, this channel must be reported empty by the sensors in all nodes of the network.

If the regulators conclude on the mechanisms to be used along with the associated parameters and thresholds, license-exempt use of secondary TVWS networks could be safely allowed. In this sense, one could deploy and operate a TVWS network anywhere and anytime, without requiring a license (just as Wi-Fi networks are currently deployed and used) and without worrying about possible interference to DTV signals. In a more controlled approach, opportunistic access could be combined with a centralized spectrum control to ensure interference free transmission to licensed users of the networks. 
DOI: https://dx.doi.org/10.26808//rs.ca.i10v2.03

International Journal of Computer Application (2250-1797)

Issue 10 Volume 2, March-April 2020

The possibility of exploiting a new business market and development of a newcommunication technology makes the research and development of TVWS really interesting.

The result of this thesis will produce available television white spaces which will be closely regulated to avoid interference among incumbent and devices utilizing them in the south eastern part of Nigeria.

\section{Review of related works}

TV white spaces (TVWSs) are frequencies made available for unlicensed use at locations where the spectrum is not being used by licensed services, such as television broadcastingspectrum. The concept of using a web based Geo location database to identify the vacant frequencies in the Television spectrum to be used for broadband access in rural areas is really reported in history with very little or no research records for the South-Eastern parts of Nigeria. Hence the need for this current work which aims at developing a web based Geo Location database capable of analyzing sensing results to make predictions as to what frequencies maybe vacant within the television spectrum at any point in time within a particular region for broadband access in the South-Eastern parts of Nigeria.Several works were researched. Some were done by:

In Fatima (2017), The Researcher adopted the vacant TV channels for secondary users. He used two different spectrum sensing techniques; the energy detector, and pilot-tone detector were employed to detect TV white space (TVWS) with minimum computational complexity. A prototype was developed using Universal Software Radio Peripheral (USRP) and designed to identify TVWS in the real time. On conducting an analysis of the obtained measurements, the results showed the state of unoccupied spectrum bands in the UHF band ranged from 500 MHz to $698 \mathrm{MHz}$ in the urban area of Windsor, Ontario, Canada. The USRP 2901 and GNU Cognitive Radio technology was used to take advantage of the underutilized spectrum to provide broadband access to secondary users in the research area. The researcher in this work concluded from their research that the presence of incumbent users can be easily detected and protected using the spectrum sensing techniques and prototype design mentioned in the thesis. The results also verified that the received signal strength of channels which were always present could be detected even when the threshold values were changed.

\section{Limitation}

In the energy detection technique used to sense the TV spectrum, frequencies with noise levels above a certain threshold could not be estimated in real time. Hence, once the value of SNR dropped below a certain threshold, the scheme could no longer efficiently detect the signals irrespective of increasing the sensing time. Also, the pilot-tone detection technique could not be implemented in cases where the receiver did not have complete information about the incoming signal. This increases the mutational complexity of his work, which led to an increase in the amount of hardware required for identifying TVWS. Hence, this technique may not be preferable for identification of TVWS in rural areas as the technique may be too expensive to implement and requires a complex hardware setup which may require maintenance from time to time.

In Ezemaet al(2015), a quantitative approach for TVWS survey was carried out on a spectral range of $470-870 \mathrm{MHz}$ within the entire campus of the Federal University of Technology Owerri in Imo State and its immediate environs, using an RF spectrum analyzer. The readings were presented using graph plots through the aid of a Touchstone PC spectrum analyzer. In this work, fixed measurement schemes were adopted and two (2) sites were chosen to take measurement. The results revealed that, $64 \%$ of the 50 channels surveyed were not in use. 
DOI: https://dx.doi.org/10.26808//rs.ca.i10v2.03

International Journal of Computer Application (2250-1797)

Issue 10 Volume 2, March-April 2020

The degree of occupancy was found to be very low, thus creating unused spectra spaces within the UHF frequency band which could be used to provide broadband services in sparsely populated rural areas.

\section{Limitation}

Although the results from the research work presented by Ezemaet al (2015) showed that a large portion of TVWS was available for opportunistic access by Secondary networks with only about $36 \%$ of the considered spectral range being occupied by licensed users as of the time the research was carried out. Nonetheless, there was no system put in place to make the results obtained from the measurements readily available for the intended users (WSDs).A database was developed to handle this limitation in the current work. It holds the results obtained from measurements carried out in the test bed area thereby allowing White Space Devices to access this information with the help of a web page that is capable of querying the database, analyzing the results and identifying TVWS.

In marcozennaro et al,(2015) it shows that although TV white space geo location database protects TV transmitters and receivers from interference sufficiently by keeping records of TV transmitters information, and relying on propagation models to determine the protection area of TV transmitters, the work tries to prove that how accurate a geo location database are in determining the protection area of TV transmitters is largely dependent on how accurate the propagation model is in predicting the TV signal attenuation (path loss) over distance. Propagation models are designed for specific type of environment and terrain, and as such their accuracy in predicting signal attenuation is largely affected by these two factors.

\section{Limitations}

The propagation models were scarcely tested in developing world where white space is assumed to provide broadband connectivity. As such, it is unclear at the moment how accurate the geo location database will detect TV white spaces in the developing world.

\section{METHODOLOGY}

This research work seeks to achieve the development of a web based geo-location data base that provides White Space Devices (WSDs) with accurate information on which frequencies are occupied and which are not within the UHF band of the Television spectrum (taking Ngwo Community of Enugu State, South-East Nigeria as case study), at any point in time and on any day of the week. A combination of software and hardware components was used as required to achieve this goal

. In this section, the experimental setup for a continuous frequency sweep of the area under observation was critically analyzed taking into consideration the sensing technique being the technique of choice for this work in determining the state of the TV spectrum. TV spectrum sweeps were carried out daily (every fifteen minutes) for a duration of one week i.e. from Monday to Sunday to be able to give an accurate representation of the active Television frequencies within the UHF band at any time of the week.

Again, the MYSQL database application and data translation technique to be used to import the scanning results from excel spread sheet format into the database is looked at, also the query language with the PHP script developed for WSDs to query the database over the internet to be able to access, analyze and predict the available TVWS within the research region was proposed.Language with the PHP script developed for WSDs to query the database over the internet to be able to access, analyze and predict the available TVWS within the research region was proposed. 


\section{HARDWARE COMPONENTS USED}

The RF Explorer is the hardware component used in this work. It is essentially a handheld digital spectrum analyzer. Its operation is based on a highly integrated frequency synthesizer which offers high performance. For this work, we used RF Explorer model 3G combo, this model comes with a Nagoya NA- 773 wideband telescopic and whip dipole antenna. The unit serves as the spectrum sensing equipment in this setup and is used to detect the active frequencies within the television spectrum of the area under observation.

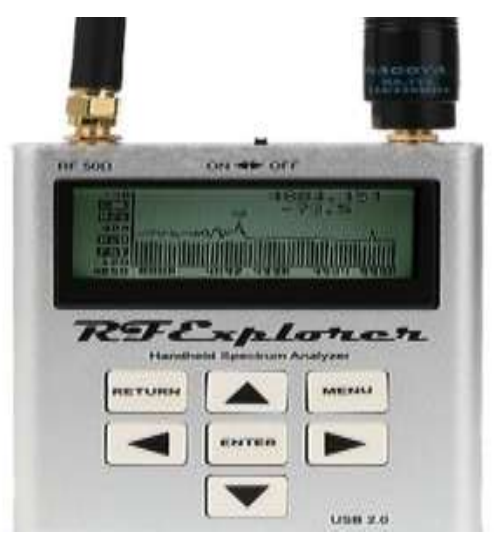

Fig 1: RF Explorer Spectrum Analyzer

\section{SOFTWARE TOOL SET}

RF Explorer Windows Client: This is the main tool for communication between RF Explorer device and PC. It display high resolution graphics of monitored RF signals in real time, it troubleshoot RF issues, and detect sources of RF interference. The main screen includes all the functionality to make Spectrum Analyzer measurements in a PC. The results from its operation can be saved and viewed in the following format; graphics, print, zoom in/out in CSV log files as a waterfall - heat map or in Microsoft excel for better interpretation.

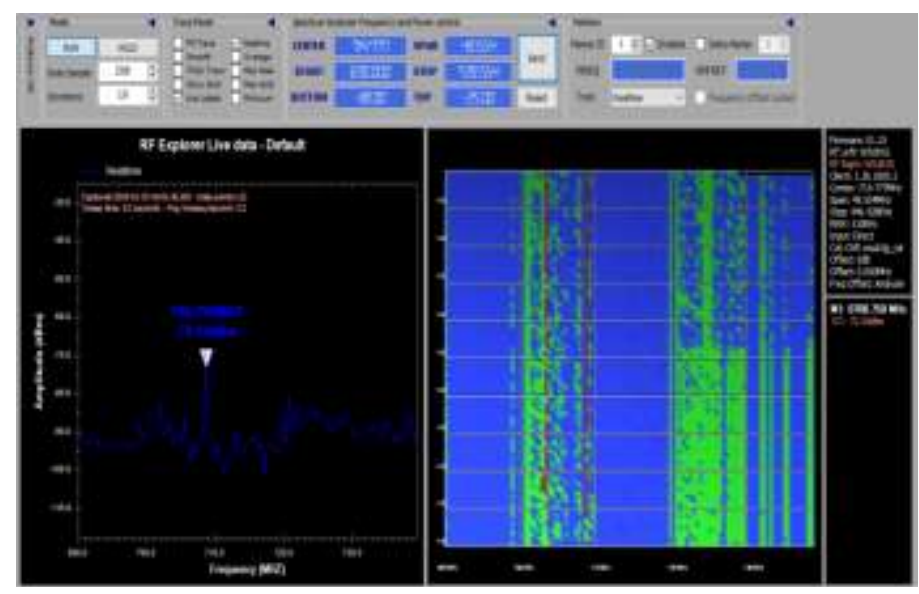

Fig 2: RF Explorer client interface

WAMP Server (MySQL Database): The WAMP Server is a Web development platform on Windows that allows you to create dynamic Web applications with Apache2, PHP, and MySQL. WAMP Server automatically installs everything you need to intuitively develop Web applications. You will be able to tune your server without even touching its setting files. 
Best of all, WAMP Server is available for free (under GPML license) in both 32 and 64 bit versions. Alongside, PHPMYADMIN allows you to manage easily your databases. WAMP server is not compatible with Windows XP, SP3, or Windows Server 2003.

PHPMYADMIN: this is a tool set or function contained in the WAMP server, this interface holds information on all the data bases contained in the array and should also possess the following qualities; Features provided by the program include: 1. Web interface 2. MySQL and MariaDB database management 3. Import data from CSV and SQL 4. Export data to various formats: CSV, SQL, XML, PDF (via the TCPDF library), ISO/IEC 26300 - Open Document Text and Spreadsheet, Word, Excel, LaTeX among others In this work, the PHPMYADMIN application was used in the administration of query on the developed database as duly required.

Adobe Dreamweaver: This is a development application that combines a visual design surface known as Live View and a code editor with standard features such as syntax highlighting, code completion, and code collapsing as well as more sophisticated features such as real-time syntax checking and code introspection for generating code hints to assist the user in writing code. Combined with an array of site management tools, Dreamweaver lets its users design, code and manage websites as well as mobile content. Dreamweaver is positioned as a versatile web design and development tool that enables visualization of web content while coding. Dreamweaver, like other HTML editors, edits files locally then uploads them to the remote web server using FTP, SFTP, or WebDAV.

Google Maps: Google Maps is a web mapping service developed by Google. It offers satellite imagery, street maps, $360^{\circ}$ panoramic views of streets (Street View), real-time traffic conditions (Google Traffic), and route planning for traveling by foot, car, bicycle, or public transportation. It also offers longitude and latitude positioning services with GPs positioning.

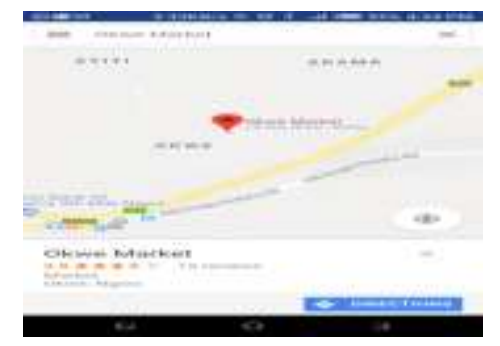

Fig 3: Google Maps Showing Measurement Location

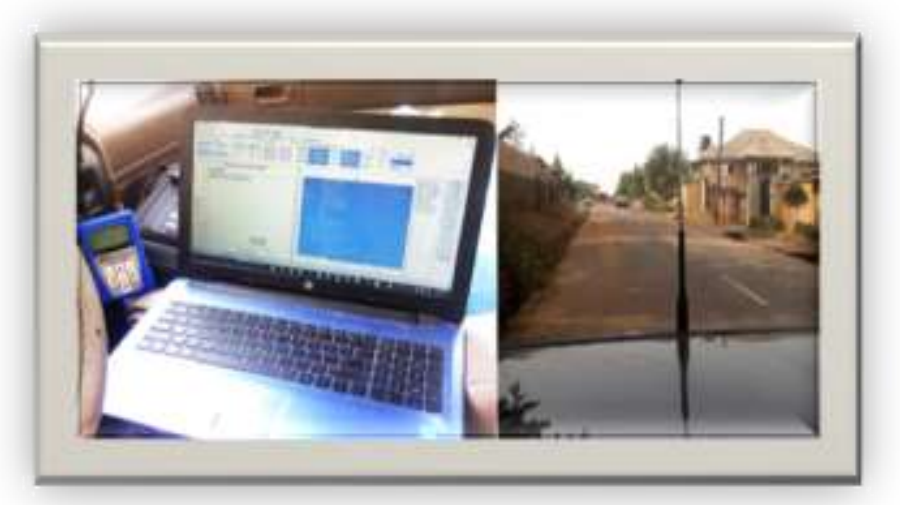

Fig 4: RF Explorer Scanning Setup 


\section{STRUCTURE FOR WEB BASED GEOLOCATION DATABASE}

The MYSQL database structure adopted for this research work was simplified for easy operation by the administrator to enable an effortless manipulation of the table data and helping with quick and effective analysis of query results. The flowchart below shows the query flow structure of the designed database for this work.

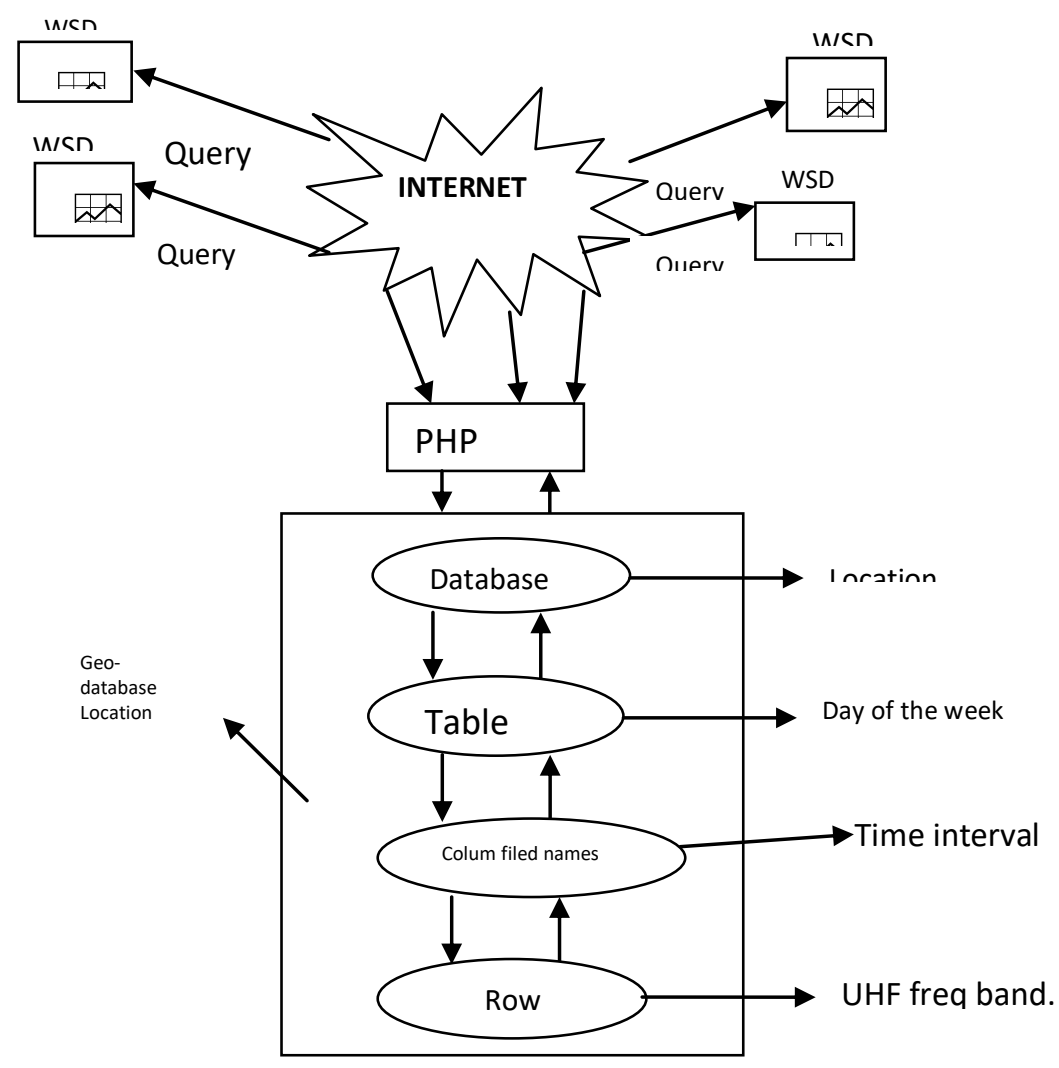

Fig 5: Structure for Web based Geo-location data base

\section{THEORY OF OPERATION}

After the scanning of the UHF band of the television spectrum scheduled every fifteen minutes for one week (Monday - Sunday) has been completed successfully using the RF Explorer, the results are carefully imported into the WAMP server. This process requires that a series of conversions is to be carried out on the scanning results which is in excel (XLSX) sheet format before it can be successfully imported in to the WAMP server environment. A Span of $20 \mathrm{MHz}$ was used to enhance sensitivity of the spectrum measured, and the frequency resolution for the experiments was set to $178.6 \mathrm{KHz}$ on the RF Explorer window client with an omnidirectional antenna fixed on top of a car and the laptop inside the car as shown in figure 5 . The antenna height is $1.5 \mathrm{~m}$ above the ground at the site under observation where frequency sweeps were carried out.

Table 1: Measured GPS Location for NGWO

\begin{tabular}{|c|c|c|}
\hline SITE NAME & LATITUDE & LONGITUDE \\
\hline Ngwo & 6.434102 & 7.451993 \\
\hline
\end{tabular}


In the WAMP server environment, the imported scanning results are grouped categorically and this grouping or structuring is the backbone of this entire Geo location database design. The database structure for this work is described below.

- The Database Name: it holds the information about the LOCATION or area where the scanning results were obtained, in this case NGWO community.

- Table Name: this identifies uniquely which day of the week the scanning results represent. For this thesis, the result for Monday's scan is represented as Monday same goes for every other day of the week.

- Column or Field Name: this is a time representation of the individual scanning carried out every fifteen minute interval starting from 12 mid night.

- Row Equivalent: these represent the corresponding row values for the frequency and its corresponding energy level at that particular time of reference.

- White Space Devices: These are the intended secondary/opportunistic users of the television white spaces lodged within the UHF band of the television spectrum.

- PHP Script

Hypertext Pre-processor Language (PHP) is a programming language used for web development. The PHP script is the written code that is responsible for the logic aspect of the entire system. It also serves as the brain of the entire setup as it handles all calculations, approximations, data manipulations, connecting to database and even oversees the activities of the database querying process executed in the webpage. The PHP scripts are embedded within the HTML elements. The HTML elements are responsible for the look and feel of the interactive interface. In other words, Hyper Text Markup Language (HTML) elements are responsible for designing the web pages which is the interacted interface for the WSDs.

- Internet

For this research, the PHP script is uploaded to a remote C-PANEL alongside the Geolocation data base that has already been properly structured. This setup enables the web page to be readily accessible to WSDs over the internet. For this purpose the internet can be viewed as an access channel to the Geo-location database which holds all the results from the frequency scan/measurements carried out.

\section{RESULT AND ANALYSIS}

The development of the web based Geo Location database in this work was segmented into stages. The first stage that was carried out was the TV spectrum sweep achieved with the help of the RF Explorer device taken at fifteen minutes intervals for one week.

\section{Table 2: RF Explorer scanned result}

\begin{tabular}{|c|c|c|c|c|c|c|c|c|c|c|c|}
\hline \multicolumn{12}{|c|}{$\begin{array}{l}\text { RF Explorer CSV data file:RF Explorer PC Client - Format v002 } \\
\text { Start Frequency: 470MHZ }\end{array}$} \\
\hline \multicolumn{12}{|c|}{ Step Frequency: 446.6KHZ } \\
\hline \multicolumn{12}{|c|}{ Total data entries: 96} \\
\hline \multicolumn{12}{|c|}{ Steps per entry: 112} \\
\hline $\mathrm{S} / \mathrm{N}$ & Time & $\begin{array}{c}\text { RSS at } \\
470\end{array}$ & $\begin{array}{c}\text { RSS at } \\
470.17 \\
9\end{array}$ & $\begin{array}{c}\text { RSS at } \\
470.35 \\
7 \\
\end{array}$ & $\begin{array}{c}\text { RSS at } \\
470.53 \\
6\end{array}$ & $\begin{array}{c}\text { RSS at } \\
470.71 \\
4 \\
\end{array}$ & $\begin{array}{c}\text { RSS at } \\
470.89 \\
3\end{array}$ & $\begin{array}{c}\text { RSS at } \\
471.07 \\
1 \\
\end{array}$ & $\begin{array}{l}\text { RSS at } \\
471.25\end{array}$ & $\begin{array}{c}\text { RSS at } \\
471.42 \\
9 \\
\end{array}$ & $\begin{array}{c}\text { RSS at } \\
471.60 \\
7\end{array}$ \\
\hline 1 & $\begin{array}{c}0: 15: 0 \\
0\end{array}$ & -93.5 & -90.5 & -95 & -92.5 & -93 & -89 & -89 & -98 & -95 & -98 \\
\hline 2 & $\begin{array}{l}0: 30: 0 \\
0\end{array}$ & -95 & -93 & -91 & -90.5 & -88.5 & -96 & -91 & -95.5 & -98 & -93 \\
\hline
\end{tabular}


DOI: https://dx.doi.org/10.26808//rs.ca.i10v2.03

International Journal of Computer Application (2250-1797)

Issue 10 Volume 2, March-April 2020

\begin{tabular}{|c|c|r|r|r|r|r|r|r|r|r|r|}
\hline 3 & $\begin{array}{c}0: 45: 0 \\
0\end{array}$ & -93.5 & -96 & -90 & -93 & -95.5 & -88 & -93.5 & -91 & -93.5 & -97.5 \\
\hline 4 & $\begin{array}{c}1: 00: 0 \\
0\end{array}$ & -90 & -100 & -98.5 & -93.5 & -91 & -96 & -93 & -94.5 & -98.5 & -93 \\
\hline 5 & $\begin{array}{c}1: 15: 0 \\
0\end{array}$ & -95.5 & -96 & -94 & -90.5 & -94 & -98.5 & -91 & -94 & -99 & -93 \\
\hline 6 & $\begin{array}{c}1: 30: 0 \\
0\end{array}$ & -94 & -90.5 & -94 & -96.5 & -90 & -97 & -98.5 & -91 & -92.5 & -93 \\
\hline 7 & $\begin{array}{c}1: 45: 0 \\
0\end{array}$ & -92.5 & -98 & -93.5 & -93 & -91 & -101.5 & -92 & -93.5 & -97 & -96.5 \\
\hline 8 & $\begin{array}{c}2: 00: 0 \\
0\end{array}$ & -91.5 & -92 & -94 & -96 & -91 & -89.5 & -89 & -95.5 & -92.5 & -98.5 \\
\hline 9 & $\begin{array}{c}2: 15: 0 \\
0\end{array}$ & -89.5 & -95.5 & -93 & -92 & -93 & -95 & -96.5 & -93 & -92.5 & -97.5 \\
\hline 10 & $\begin{array}{c}2: 30: 0 \\
0\end{array}$ & -95 & -96 & -95 & -91 & -96 & -88 & -93 & -91 & -96.5 & -99.5 \\
\hline
\end{tabular}

In the next stage, a web page interface was developed using HTML tags and the PHP code snippets to handle the logic flow of the entire system. This stage is essentially the brain of the entire setup as it handles the querying of the database by the WSDs, identification of TVWS from amongst the sweep results and TV spectrum predictions deduced from sweep history. This stage is also responsible for facilitating the connection between the WSD, Web Page and the data base. For this work, the web page is subdivided into three essential pages. The first page or home page displays an interactive interface. Here, the WSD fills the required fields displayed on the interface before it is allowed to query the database successfully. Once completed and sent, the webpage automatically uses the imputed information to query the data base and displays the results in the result page in a matter of seconds.

The query result page displays the results generated by the query page simply by securing a connection to the database where the sweep results are were structured and stored. The related information is selected and automatically analyzed by the PHP codes before displaying results as shown in fig:8

\section{WEB-BASED GEO-LOCATION DATABASE FOR TV WHITE SPACE DEVICES (TVWSDs).}

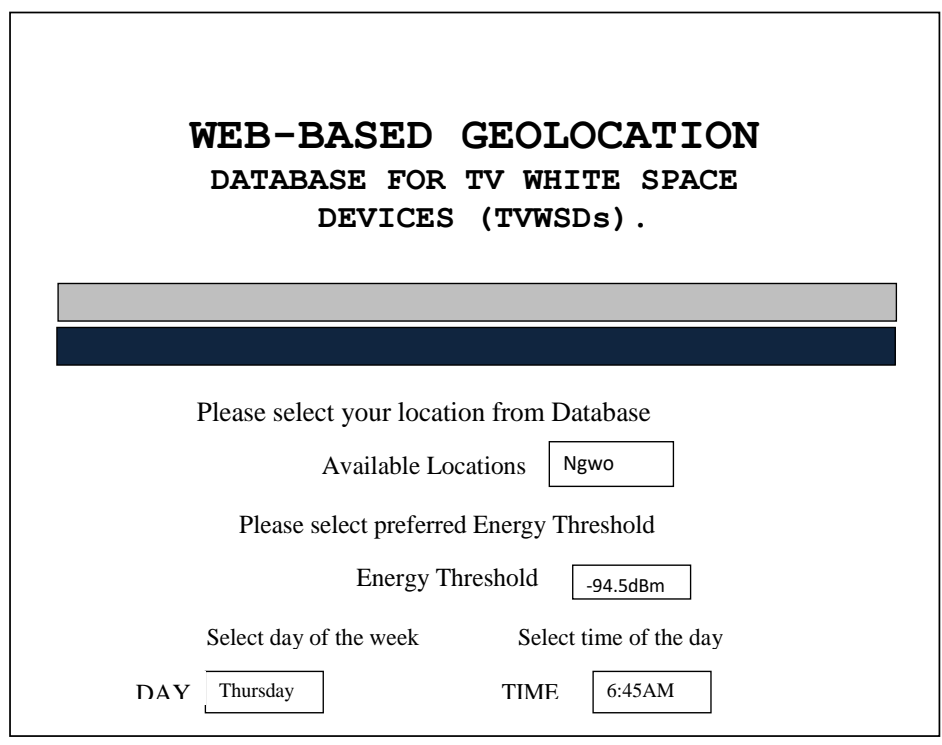

Fig 6: TV white space finder (Query page) 
Table 3:Spectrum availability as of 12th 0f October, 2019

\begin{tabular}{|l|l|}
\hline \multicolumn{2}{|c|}{ SEARCH VARIABLES } \\
\hline LOCATION & Ngwo \\
\hline DAY & Friday \\
\hline TIME & $12: 13$ \\
\hline THRESHOLD & $-94.5 \mathrm{dBM}$ \\
\hline Height above Ground & $1.5 \mathrm{M}$ \\
\hline
\end{tabular}

\section{Table 4: Query Result Page}

UHF Band Search Result Analysis

\begin{tabular}{lc} 
Occupied Freqs(MHz) & 374 \\
Vacant Freqs(MHz) & 410 \\
Total Freqs(MHz) & 784 \\
Percentage Use(\%) & $47.704 \%$ \\
\hline Percentage Free $(\%)$ & \\
\hline Vacant Frequencies & $52.296 \%$ \\
\hline $440 \mathrm{MHz}$ & Energy Levels \\
\hline $440.893 \mathrm{MHz}$ & $-97 \mathrm{dBmfree}$ \\
\hline $441.339 \mathrm{MHz}$ & $-98 \mathrm{dBmfree}$ \\
\hline $442.232 \mathrm{MHz}$ & $-98.5 \mathrm{dBmfree}$ \\
\hline
\end{tabular}

The display on the left illustrates the spectrum occupancy of the TV spectrum obtained from the last sweep results updated to the database. It shows the query or search variables imputed by the WSD used to generate the displayed results. It also shows the result analysis with amount of occupied, vacant frequencies, percentage of used and vacant frequencies respectively. Finally, a bar chart is generated to further illustrate the disparity between amounts of occupied to vacant frequencies as at the time the sweep was carried out. The display to the right of the screen is a generated prediction of what the TV spectrum occupancy would be as at the current day and time of the query based on the TV spectrum history gathered over the one week TV spectrum sweep exercise. The vacant frequencies generated in the frame on the right were enabled to be clickable such that the WSD can select one of the predicted vacant frequencies displayed in the bar chart. Once a vacant frequency is selected by the WSD, the frequency history page is initiated and a frequency history or timeline is generated showing the sensed energy levels of the selected frequency at that particular reference time for the entire week. It also pulls up from the database the energy levels recorded fifteen minutes before and after the reference time for the same frequency 
DOI: https://dx.doi.org/10.26808//rs.ca.i10v2.03 International Journal of Computer Application (2250-1797) Issue 10 Volume 2, March-April 2020

under observation. This gives the WSD a better understanding of the occupancy history of the selected frequency. Finally, the predicted energy level is displayed along with the allowable transmission power for that frequency

Table 5: Frequency History Page FREQUENCY HISTORY FOR (449.375 MHz)

\begin{tabular}{|c|c|c|c|c|c|c|c|}
\hline ReferenceTime & $\begin{array}{c}\text { MON } \\
(8 / 10 / 18)\end{array}$ & $\begin{array}{c}\text { TUE } \\
(9 / 10 / 18)\end{array}$ & $\begin{array}{c}\text { WED } \\
(10 / 10 / 18)\end{array}$ & $\begin{array}{c}\text { THR } \\
(11 / 10 / 18)\end{array}$ & $\begin{array}{c}\text { FRI } \\
(12 / 10 / 18)\end{array}$ & $\begin{array}{c}\text { SAT } \\
(13 / 10 / 18)\end{array}$ & $\begin{array}{c}\text { SUN } \\
(14 / 10 / 18)\end{array}$ \\
\hline $15 \min$ Before & $\begin{array}{c}- \\
97.5 \mathrm{dBM}\end{array}$ & $\frac{-}{103 \mathrm{dBM}}$ & $-96.5 \mathrm{dBM}$ & $-93.5 \mathrm{dBM}$ & $-96.5 \mathrm{dBM}$ & $-97.5 \mathrm{dBM}$ & $-94.5 \mathrm{dBM}$ \\
\hline $12: 13$ & $\begin{array}{c}- \\
96.5 \mathrm{dBM}\end{array}$ & $-95 \mathrm{dBM}$ & $-94 d B M$ & 98.5 $\overline{-}$ dBM & $-94 \mathrm{dBM}$ & $-97 d B M$ & 96.5 $\overline{-}$ \\
\hline 15min After & $\begin{array}{c}- \\
97.5 \mathrm{dBM}\end{array}$ & $\begin{array}{c}- \\
103 \mathrm{dBM}\end{array}$ & $-96.5 \mathrm{dBM}$ & $-93.5 \mathrm{dBM}$ & $-96.5 \mathrm{dBM}$ & $-97.5 \mathrm{dBM}$ & $-94.5 \mathrm{dBM}$ \\
\hline
\end{tabular}

Average Energy Level For Today 2019-05-03 (-94.9 dBM) AllowableTx Power (4W)

\section{VII.CONCLUSION}

This research work aims to achieve the development of a web based Geo-location database for predicting/finding television white spaces in Ngwo environment in Enugu, south East Nigeria. The frequency sweep of the UHF band of the television spectrum was measured (i.e. from $470 \mathrm{MHz}$ to $789 \mathrm{MHz}$ ) using the spectrum sensing technique and was taken at intervals of 15 minutes for one week while the results were structured into a database. A webpage user interface was developed and hosted over the internet. White space devices can access this web page via the unique web address and using the query options on the homepage/query page, they are able to query the database. The result page in turn automatically generates an analysis based on the query options sent to the database by the WSD. The analysis generated by the query page helps identify which frequencies are occupied by a primary user and which frequencies are vacant for secondary use. The analysis calculates and displays the amount of used and unused frequencies as well as the percentage of used frequencies to the unused frequencies at any point in time of the week per selected location. Finally from the analysis, a prediction is made on TVWS availability at that reference time based on spectrum records stored in the Geo location database. The analysis generated is uniquely different and greatly depends on the Location, day of the week, energy threshold and time selected by the WSD from the query page or home page. From result analysis generated, it was gathered that for a standard energy threshold of $-94.5 \mathrm{dBM}$ specified by FCC, more than $65 \%$ of the frequencies in the UHF band of the television spectrum is left unused at any time of the day and on any day of the week in Ngwo community of Enugu State, South East Nigeria. This result goes to show how the current fixed spectrum assignment to large geographic area policy has proved to be an uneconomical and inefficient use of the rather limited radio resource especially with the current explosion in the demand for spectrum due to the evolution of broadband hungry applications such as face book, twitter and their likes. Furthermore, the Geo-location database developed in this work also promises to totally eradicate the probability of interference between primary and secondary users within the UHF band if only the fidelity of the database is not compromised hence, TV spectrum sweep or scan should be carried out as frequently as possible to ensure that the information represented in the data base is as up to date and as accurate as possible reflecting the current state of occupancy of the TV spectrum. Finally, 
DOI: https://dx.doi.org/10.26808//rs.ca.i10v2.03 International Journal of Computer Application (2250-1797) Issue 10 Volume 2, March-April 2020

from the generated results obtained from the result page, it can be concluded that there is a great potential to provide broadband internet access over TV white space frequencies in Ngwo community of Enugu State as well as the entire south east since there's an excess of more than $65 \%$ of TV white space available at any time of the day/week. In general, the TVWS technology proves to be a step in the right direction in solving the last mile problem in rural areas of Nigeria.

\section{REFERENCE}

1. Ageng'o. C (2014)," Mydigitalbridge Foundation, Microsoft plop whitespace project brings

2. Broadband to Namibia", Techweez, retrieved December 6, 2014.

3. Akylidiz IF, W.Y. Lee, M.C, Vuran, and S, Mohanty (2006), "Next Generation Dynamic spectrum Access/Cognitive Radio Wireless Networks.A Survey", Science Direct, Computer Networks, Vol.50, No. 13, pp 2127-2159.

4. Alemu and Tizazu, (2012)."Spectrum Availability Assessment Tool for TV White Space," in School of Electrical Engineering Department of Communications and Networking Technologies Professorship: Communication Engineering.

5. Alexander, Wyglinski .M, Maziar .N,\&Thomas.Hou .Y, (2010) "Cognitive Radio Communications and Networks: Principles and Practice". Burlington MA: Academic, Pg.: 39-40.

6. Anderson .K, (2016). "Using TV White Spaces for Rural Broadband," CarlsonWireless Technologies. Retrieved fromhttp://www.carlsonwireless.com/products/ruralconnectip.htm

7. Carlson \&Telcordia Company, (2015) '"How TV White space devices work,' Submitted to the University of Texas USA, Pg: 121-125.DARPA XG WG, (2003). retrieved on May 26, 2017.

8. .Farzad, H. (2013) "capacity considerations for secondary networks in TV White Space.

9. Ezema, (2015) A survey on the availability of TVWS in Eastern Nigeria (FUT Owerri) Dec. 2015.

10. Faruk, (2013) Digital television coverages and protection contour estimation for spatial white space.

11. .Farzad, H. (2013) "capacity considerations for secondary networks in TV White Space.

12. Fatima, (2017) Real time implementation of spectrum sensing Technology in cognitive radios. Department of electrical and Computer engineering Windsor Canada

13. GarimaMaheshwari and Animesh Kumar (2015). "Optimal Quantization of TV White Space Regions for a Broadcast Based GeolocationDatabase.”. Department of Electrical and Engineering Indian Institute of Technology, Bombay Mubai, India.

14. Gaurang, Animesh .K, Abhay.K,(2013)“"Towards Enabling Broadband for a Billion Plus population With TV White Spaces", Department of Electrical Engineering Indian Institute of Technology Bombay Mumbai 400076 India.

15. Ghasemi .A, Sousa .E.S, (2005).“Collaborative spectrum sensing for opportunistic access in fading environment", In Proc. IEEE DySPAN .

16. Imbriglio, (2016) Coperative spectrum sensing for cognitive radio: performance analysis for Realistic system setups and channelconditions university of Aquila, department of Electrical and information Engineeering Italy.

17. Kassem Mohamed, (2018) On the potential of TVWS spectrum to enable a middle mile network infrastructure, school of information university of Edinburgh UK.

18. Liu .T, (2009). "Analytical Modeling of HSUPA-Enabled UMTS Networks for 
DOI: https://dx.doi.org/10.26808//rs.ca.i10v2.03 International Journal of Computer Application (2250-1797) Issue 10 Volume 2, March-April 2020

19. Capacity Planning," Doctoral dissertation submitted to the School of Information Technologies at the University of Sydney.

20. Mac Bowel (2015), "Primary contour prediction Based on Detailed Topographic Data and its impact on TV White space availability Bagula and S. NLEYA,

21. Marco Zennaro. (2015) "On the Relevance of Using Affordable tools for White Space Identification," in proceedings of the IEEE $8^{\text {th }}$ International Conference on Wireless and Mobile Computing, Networking and Communications. (WiMob), pp 606-611.

22. Maier .M, Ghazisaidi .N and Reisslein .M, (2008)."The Audacity of Fiber-Wireless (FiWi) Networks (Invited Paper)," Proceedings of ICST International Conference on Access Networks.pp.16 - 35.

23. Mansi and Gajanan (2011).A survey in spectrum sensing technique in cognitive radio Networks.Department of Electronics and Telecommunication Engineering Navi Mumbai India.

24. Mike Harwood,(2009). "Network+ Exam Cram: Wireless Networking" retrieved from http://www.pearsonitcertification.com/articles/article.aspx?p=1329709\&seqNum=4.

25. MaziarNekovee, (2010) “,A survey of cognitive radio access to TV white spaces, International Journal of Digital Multimedia Broadcasting. /"

26. http://www.quasarspectrum.eu/images/stories/Documents/publications/Nekoveejournal2010.pdf

27. Naroa.Z, (2011) 'Cognitive Radio and TV White Space Communications,' in2011. IEEE Journal on Wireless Communication and Networking, Pg.: 19.

28. Opawoye, Faruk .N, Bello .O.W \&Ayeni .A .A, (2015)."Recent trends on TV White space deployments in Africa," Department of Telecommunication Science, University of Ilorin, Kwara. Nigeria.

29. Seabold.D.(2011), “UK Leads Europe in White Space Technology”, EU Design studio, retrieved on December 4, 2017.

30. Simba .F, (2010). "Modeling Connectivity for e-Learning in Tanzania: Case-Study of

31. Rural Secondary Schools," Licentiate Dissertation, Sweden.

32. Simba .F, Mjema .E.M, (2011). "Broadband Access Technologies for Rural

33. Connectivity in Developing Countries" retrieved from International Journal of Research and Reviews in Computer Science (IJRRCS) Vol. 2, Pg 2,

34. Tang (2017) "cooperative spectrum sharing in cognitive radio Networking”.Department of Electrical and Computer Engineering waterloo, Ontario Canada.

35. Timothy, Bello .O.W, \&Karandikar, (2014). "The potential for use of TV white spaces for the internet in Zimbabwe" IEEEconference paper .

36. Tkachenko .A, Cabric .D, and Brodersen .R .W, (2007) "CyclostationaryFeature Detector Experiments using Reconfigurable BEE2," In Proc. IEEE Int. Symposium on New Frontiers in Dynamic Spectrum Access Networks, Dublin, Ireland, pp. 216-219.

37. Won-Yeol .L, Akyildiz .I.F, Mehmet .C, Vuran, Shantidev .M, (2006)."NeXT generation/dynamic spectrum access/cognitive radio wireless networks: A survey", Broadband and Wireless Networking Laboratory, School of Electrical and Computer Engineering, Georgia Institute of Technology, Atlanta, GA 30332, United States"Wireless innovation alliance". Web site, retrieved November 20, 2017.

38. Yang .J, (2004). "Spatial channel characterization for cognitive radios," Master's thesis, University of California, Berkeley, Pg.: 24-26.

39. Yinusa, AAdediran (2014). "TV White Space in Nigeria in UHF Band: Geo- spatial Approach" 